\title{
Intelligent Wireless Environmental Monitoring System of University Laboratory Based on Internet of Things
}

\author{
Jiang Wenwen, Zhai Linbo", Zhang Feifan, Gong Wenjing, Gong Yuxin \\ School of Information Science and Engineering, Shandong Normal University, Jinan, China. \\ *corresponding author: zhai@mail.sdu.edu.cn
}

Keywords: university laboratory, environmental monitoring, single chip microcomputer, wireless communication

\begin{abstract}
The system is mainly used in university laboratories. The main purpose we design this system is to monitor the data of laboratory environment timely and improve the efficiency of laboratory inspection. The system mainly includes three parts: the front data acquisition, wireless communication and security alarm. The single chip microcomputer is the core of this system. The system is integrated with GPRS wireless communication and sensor function module, therefore, it can realize the data monitoring and short message warning. After many tests, the function of front-end data acquisition can be realized normally, not only the data transmission is reliable but also the alarm message is received in a timely manner. By modifying the function module, the system can also be applied to other environmental monitoring, which has a certain practical and promotional value.
\end{abstract}

\section{Introduction}

Environmental monitoring is one of the key points of the present university laboratory management work. It has high reliability to ensure the laboratory safety by using manual inspection[1], but it wastes manpower and time in this way. Using traditional wiring experiment alarm device (such as $R S$ - 232 ) [2] could save more resources comparing with manual inspection. But it is not conducive to popularize and use because of the restriction of conditions, such as wiring environment and transmission distance. Using the ZigBee wireless data transmission network[3] is easily restricted by conditions like transmission distance. Short messages are easily congested if using GSM dial-up way of circuit-switched data transfer, so it may leads to short messages losing. Compared with the above data transmission ways, using GPRS communication of packet switching technology can realize reliable remote wireless communication. The proposed system of this article is a kind of Internet of things application. It puts forward a design idea of the intelligent wireless monitoring with the characteristics of high cost performance, user-friendly control and reliable operation. Compared with traditional environmental monitoring mode, this system has stronger pertinence to environmental monitoring and it is favorable for promotion use. It can also speed up the information construction of colleges and universities.

The first part of the article introduces the overall design and implementation of the system. It mainly makes an introduction for the function of system, the design and implementation of the model. The second part could make overview of control, communication module and data acquisition module. The third part introduces the system software design idea, the testing method of hardware 
and software. It has also made the example analysis to some debugging results. The fourth part of the article summarizes the design ideas and design methods of this system.

\section{System overall design and implementation}

\subsection{System function introduction.}

Through the objective analysis of the present Internet of things technology development and the status of the university laboratory management, we integrate wireless communications, sensor technology, embedded and other important technologies [4], develop a monitoring system. And this system integrates various functions, such as temperature and humidity detection, monitoring indoor light off, anti-theft, fire prevention and wireless communications. The main functions of the system are as follows:

- Testing the concentration of laboratory combustible gas 24 hours a day and investigate fire risk.

- Achieving the multiple-point measurement of temperature and humidity, and feedback the measured data of temperature and humidity accurately.

- Monitoring whether there are illegal personnel to enter the laboratory during the non-use period, to achieve effective guard against theft.

- Monitoring light intensity during the non-use period in the laboratory, judge and return the situation of lighting of the laboratory.

- Wireless communication in time. When the disaster occurs, the system on-site start buzzer alarm, remote dial-up alarm and inform administrators in the form of SMS at the same time.

\subsection{System Model Design and Function Realization.}

In order to achieve the above functions, the overall design of the system integrates STC12C5A60S2, GPRS module and digital sensor detection modules [5]. We use the software Protel99SE to design each part of the system hardware circuit, and produce system function development board. System architecture is shown in Figure 1.

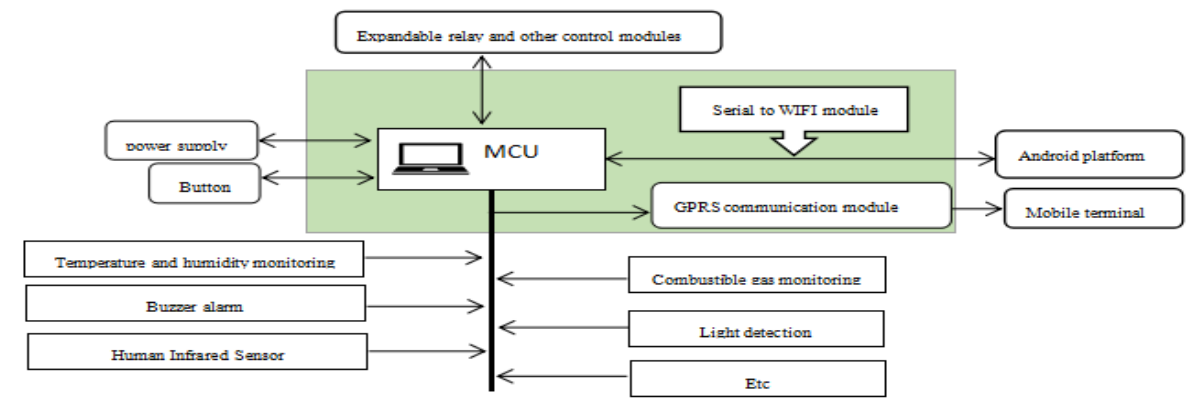

Figure 1. System architecture

Each sensor module is responsible for collecting the laboratory data, returning the data to the lab manager's mobile terminal by message. Meanwhile, the system can analyze dangerous situations (fire protection and security) when collecting data, realizing buzzer alarm and SMS alarm automatically. In addition, system development board takes automatic data acquisition, SMS command control and data control into account when designed, so the system integrates the serial port to WiFi module and can be programmed through the JAVA program based on Andorid system[6], achieving data detection and remote control through mobile terminal APP.

With the design and establishment of large scale laboratory management system, the system can 
be used independently, it can also be treated as a subsystem to connect the public communication network through the specific interface, transmitting the sensing information to the Internet of things center server. Then, the platform analyze and process the collected information, so the client can use different terminals to access and control.

\subsection{Each module instructions of system}

\subsubsection{Control and communication module.}

The system chooses a new generation of single chip microcomputer STC12C5A60S2 as control core. It uses GPRS module, in the form of SMS, completes the communication with the mobile terminal to facilitate real-time monitoring.

The main function of GPRS module is to initialize to electricity and create a thread into the listening state after the serial port connected to MCU. When the GPRS data sending thread monitors the buffer when data is written in, it will send the collected data to the designated mobile phone terminals. This system design chooses SIM900A module embedded TCP/IP protocol. The expanding TCP/IP AT commands is convenient for users to use TCP/IP protocol and transmit data. We can use the AT command to control GPRS module. By querying the corresponding command the user manual, we can know that the AT command mainly consists of initialization, set the data format, waiting to send, etc.

The remote communication framework design of the system will build a system communication mode based on STC12C5A60S2 and GPRS network. As shown in Figure 2, when a user sends query request to center control unit in the form of mobile phone short message, MCU detects the information to user's request through GRPS network access point.

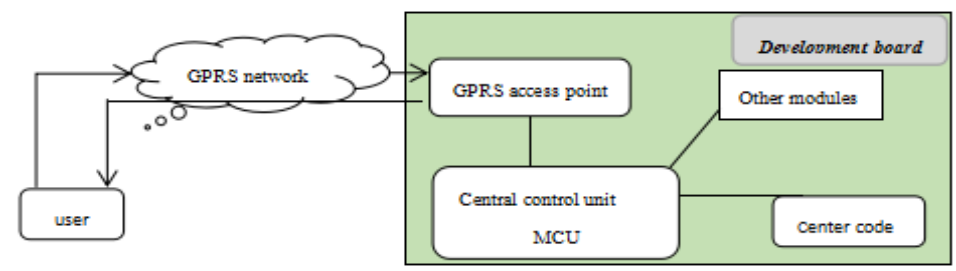

Figure 2. Design of System Telecommunication Frame

\subsubsection{Data acquisition module.}

System acquisition module chooses digital sensors without external A/D conversion, it is more convenient to design it. In order to ensure the normal order of the GPRS module launch, the development board adopts DC12V / 2A power for supply. $3.3 \mathrm{~V}$ and $5 \mathrm{~V}$ voltage chip are built-in. It can protect the other data gathering module to work normally. The selection of data acquisition sensor module is shown in Table 1.

Table 1 The Selection of Data Acquisition Sensor Module

\begin{tabular}{|c|c|}
\hline Module & Model \\
\hline $\begin{array}{c}\text { Temperature and humidity acquisition } \\
\text { module }\end{array}$ & DHT11 \\
\hline Fire monitoring module & MQ-9 \\
\hline Light intensity acquisition module & GY-30 \\
\hline Infrared sensor module & HC-SR501 \\
\hline
\end{tabular}




\subsection{System software design}

The system mainly uses the Keil-uVision4, computer, Proteus, Protel99SE and the firing machine as the development tool. It combines system control instruction, wireless communication protocol and the sensor use manual to design the module function of system.

Build project in Keil-uVision4's C language development environment [7], design and achieve the source code of the system module function. Use Proteus and Keil-uVision4 to realize the module simulation in the stage of the hardware circuit design. Prote199SE is used as a design tool to complete hardware circuit schematic and PCB board. Finally confirm that all the source code debugging is correct, burn the program after partial hardware circuit simulation is correct.

\section{System test}

\subsection{Program debugging method.}

The system uses the Keil-uVision4 integrated development environment to write and debug the designed program. When debugging in this environment, set program break-point at first. Break-point will be activated after start up debugging. Breakpoints can be set to conditional expression, variable, or memory access. Debugger commands or debugging functions can be executed after the break-point is triggered. And we can quickly browse the property box about the break-point settings and the location of the source program.

\subsection{Hardware testing method.}

Hardware testing requirements of this system: Testing the design of the power is the first step. Then, testing whether it can successfully start the GPRS module is the second step when providing power supply for development board. Thirdly, testing whether the various sensors can successfully collect environmental data and return to the display terminal. Fourthly, testing normal collection and data transmission of system about laboratory temperature and humidity or indoor light intensity, as well as danger tips which will be sent out when the temperature and humidity of laboratory (high requirements about temperature and humidity) which has large servers under abnormal conditions. Fifthly, testing whether there is a message response to dangerous situation when a person is walking around in the range of the human body infrared module induction. And sixthly, testing in the case of simulating fire, whether the buzzer alarm normally and whether the SMS alarm function can be achieved. At last, we integrate the whole system and select a number of laboratories for practical application testing. Then we monitor the stability of each module in the operation of the system, analyze and confirm the logical function of each module combined with the specific operation results, ensure that the system is able to carry out effective environmental monitoring and alarm. The screen-shots of system alarm SMS results are shown in Figure 3.

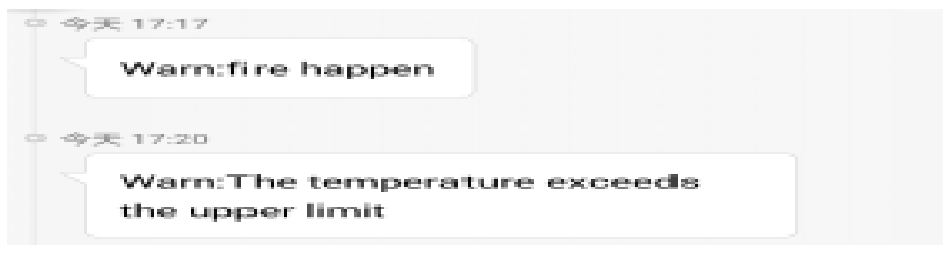

Figure 3. System alarm SMS screen-shots 


\section{Summary}

This paper outlines a design method of wireless environment monitoring system based on Internet of things in laboratory, and expounds the design idea of the system and the realization way of key functional modules. The system integrates the sensor technology, embedded technology, wireless communications and other important technologies. Lots of tests have proven that the system can achieve an effective detection of temperature and humidity, anti-theft, fire, indoor lighting monitoring off, wireless communications, SMS alarm and other functions. On the one hand, it can be extended to achieve other functions according to actual requirements. On the other hand, it can also be treated as a subsystem to access large-scale laboratory management system [8], which is cost-effective, efficient and practical, widely applicable, and achieves the expected design effect. With the continuous progress and development of technology, Internet of things technology and intelligent wireless environment monitoring technology will closely follow the pace of development of the times. With the perfect combination of theory and practice, it will help products become more cost-effective, improve the safety factor of laboratory and speed up the pace of the information construction of the university laboratories.

\section{Acknowledgements}

This work was supported by the Shandong Provincial Natural Science Foundation (BS2015DX003).

\section{References}

[1] Li Jin, Sun Wei. Quality Inspection on Environment Assessment Laboratory[A]. Environmental Science and Management. Vol.38 No.9:153-155 (2013).

[2] Kang Ping, Song Xiaoshan, Wang Luoxin,Yuan Hongchao. Serial Communication in the Intelligent Home Network[A]. Journal of Northwest University(Natural Science Edition). Vol.35 No.4:338-391 (2005).

[3] Guo Xiaodan. Surveillance and Safety System Development Based on Internet of Things[A]. Researsh and Exploration in Laboratory. Vol.33 No.4:281-285 (2014).

[4] Xu Feng, Liu Xin, Fang Baojia. Design of Smart Home Remote Control System[B]. Modern Building Electricity.No.4:21-24 (2009).

[5] Jin Ziyu, Zhang Shengchuan, Fang Maojin, Luo Gengrong. Researsh and Implemention of Smart Home System[B]. Computer Application. 47-50 (2011).

[6] Huang Ying.Laboratory Environmengal Monitoring System Based on Android Phone[A]. Researsh and Exploration in Laboratory. Vol.33 No.10:91-93/128 (2014).

[7] Zhang Peiren.The Principle and Application of MS-51 Based on C Language[M]. Programming Tsinghua University Press.2003.1.

[8] Che LinLin, Kong Yinghui, Zhao Jianli, Cheng Wenqing. Design of Wisdon Laboratory Based on Internet of things[A]. Experimental Technology and Management. Vo.30 No.10:212-215 (2013). 